\title{
CONGRUENCES FOR THE RAMANUJAN FUNCTION AND GENERALIZED CLASS NUMBERS
}

\author{
BERNHARD HEIM
}

\begin{abstract}
The Ramanujan $\tau$-function satisfies well-known congruences modulo the so-called exceptional prime numbers $2,3,5,7,23,691$. In this paper we prove new congruences related to the irregular primes 131 and 593, involving generalized class numbers. As an application we obtain distribution results. We obtain a new proof of the famous 691 congruence and congruences of the related Rankin L-funtion.
\end{abstract}

\section{IntRoduction AND STATEMENT OF RESUltS}

The Ramanujan numbers [1] $\tau(1)=1, \tau(2)=-24, \tau(3)=252, \tau(4)=-1472 \ldots$ satisfy certain famous well-known congruences modulo powers of the primes $2,3,5$, 7,23 and 691 , e.g.,

$$
\tau(n) \equiv \sum_{d \mid n} d^{11} \quad(\bmod 691)
$$

Here we prove that the Ramanujan numbers also satisfy congruences with generalized class numbers discovered by Cohen [1]: $3 H(11,3)=-3694,2 H(11,4)=$ $-50521, \quad H(11,7)=-9006448, H(11,8)=-36581523 \ldots$. These congrunces are modulo the irregular prime numbers 131 and 593. Our motivation for finding congruences of this new type was the hope of proving by congruence considerations Lehmer's conjecture that $\tau(n)$ never vanishes. As it turns out, this approach is fruitful, but in an unexpected sense.

Fundamental for the modern theory of modular forms is Deligne's [2] proof of the Serre conjecture on the existence of $l$-adic Galois representations $\rho_{l}$ attached to a modular form. In 1972 Swinnerton-Dyer determined the possible images of $\widetilde{\rho}_{l}$, the reduction of $\rho_{l} \bmod l$ for level one. This was generalized by Ono 9 .

There exist only finitely many primes $l$ such that the image of $\widetilde{\rho}_{l}$ does not contain $S L_{2}(\mathbb{Z} / l \mathbb{Z})$. These primes are exceptional and yield congruences. It is striking that the full set of exceptional primes for the modular form related to $\tau(n)$ is just the set $\{2,3,5,7,23,691\}$. This suggests finding congruences by looking at small images of the reduced Galois representation. For example, Ono [9] notes that: The search for congruences depends on the classification of subgroups of $G L_{2}(\mathbb{Z} / p Z)$, where $G L_{2}(\mathbb{Z} / p Z)$ is the full image of the representation if $l$ is not exceptional. This is certainly correct. And there is another interesting issue. The prime 691 is special in the sense that it is also an irregular prime, which gives a connection to Bernoulli

Received by the editor November 13, 2007 and, in revised form, January 9, 2008.

2000 Mathematics Subject Classification. Primary 11F33, 11F67, 11F80; Secondary 11 Y70. 
numbers, Kummer's approach to Fermat's Last Theorem, and the congruences observed here.

Our goals are threefold. We give a new proof of the 691 congruence (1.1), which as an application involves a congruence for the Rankin $L$-function $\sum_{n=1}^{\infty} \tau(n)^{2} n^{-s}$ at the critical value $s=22$ (Zagier $([13])$ ).

Theorem 1.1. Let $\Delta(z)=\sum_{n=1}^{\infty} \tau(n) q^{n}$ be the discriminant function with Fourier coefficients $\tau(n)$ and $\|\Delta\|$ the Petersson norm of $\Delta$. Then

$$
-\frac{\pi}{2 \cdot 11 \cdot \zeta(11) \cdot \zeta(22) \cdot\|\Delta\|^{2}} \sum_{n=1}^{\infty} \tau(n)^{2} n^{-22}
$$

is a quadratic residue mod 691 and

$$
12 ! \cdot B_{12} \frac{\sum_{n=1}^{\infty} \tau(n)^{2} n^{-22}}{\pi^{11} \cdot \zeta(11) \cdot\|\Delta\|^{2}} \equiv 2^{26} \cdot 3^{2} \cdot 11 \quad(\bmod 691)
$$

Also, new congruences for $\tau(n)$ are presented, related to the irregular prime numbers 131 and 593, which involve the generalized class numbers $H(t, N)$ of Cohen. Here $t \in \mathbb{N}$ and $-N$ is the discriminant of a binary positive semi-definite quadratic form. For $t=1$ these are the classical class numbers discussed by Kronecker, Hurwitz and others. Finally, numerical data illustrates the results related to $H(t, N)$.

The Ramanujan $\tau$-function $\tau: \mathbb{N} \longrightarrow \mathbb{Z}$ is defined by the Fourier coefficients of the discriminant function $\Delta$ on $\mathfrak{H}:=\{z \in \mathbb{C} \mid \operatorname{Im}(z)>0\}$ :

$$
\Delta(z):=x \prod_{m=1}^{\infty}\left(1-x^{m}\right)^{24}=\sum_{n=1}^{\infty} \tau(n) x^{n} \quad\left(x=e^{2 \pi i z}\right)
$$

It is imbedded in the family of power series of type

$$
\left\{(1-x) \cdot\left(1-x^{2}\right) \cdot\left(1-x^{3}\right) \cdot \ldots\right\}^{\alpha}
$$

where $\alpha$ varies. For example $\alpha=-1$ gives the partition function and $\alpha=24$ the discriminant $\Delta$. Many properties of $\tau(n)$ are elaborated in Ramanujan writings. He made several conjectures which have profoundly influenced mathematics. For example, he conjectured that $\tau(n \cdot m)=\tau(n) \cdot \tau(m)$ with $m, n$ coprime and that $\tau\left(p^{l}\right)=\tau\left(p^{l-1}\right) \tau(p)-p^{11} \tau\left(p^{l-2}\right)$ for all primes $p$ and integers $l \geq 2$. This is equivalent to the assertian that the Dirichlet series attached to $\Delta$ has an Euler product:

$$
\sum_{n=1}^{\infty} \tau(n) n^{-s}=\prod_{p} \frac{1}{\left(1-\tau(p) p^{-s}+p^{11-2 s}\right)}
$$

Here the product runs over all primes $p$ and $s \in \mathbb{C}$ with real part of $s$ large enough. This was proven by Mordell and was the starting point of Hecke theory, a fundamental tool in the theory of automorphic forms. The point is that the values $\tau(p)$ determine the discriminant function completely. 
Furthermore, Ramanujan predicted that the roots $\alpha_{p}$ and $\beta_{p}$ of the polynomial in the denominator of (1.6) are complex conjugates, which is equivalent to the estimate $|\tau(p)| \leq 2 p^{\frac{11}{2}}$. This was proven by Deligne as a consequence of his proof of the Weil conjectures. But there are still open questions, such as the Lehmer conjecture, that $\tau(n) \neq 0$ for all $n \in \mathbb{N}$. The following table provides some information, at the level of examples:

\begin{tabular}{|c|r|c|c|c|c|}
\hline$n$ & $\tau(n)$ & $n$ & $\tau(n)$ & $n$ & $\tau(n)$ \\
\hline \hline 1 & 1 & 11 & 534612 & $\ldots$ & $\ldots$ \\
\hline 2 & -24 & 12 & -370944 & 100 & 37534859200 \\
\hline 3 & 252 & 13 & -577738 & 101 & 81742959102 \\
\hline 4 & -1472 & 14 & 401856 & 102 & 41767088832 \\
\hline 5 & 4830 & 15 & 1217160 & 103 & -225755128648 \\
6 & -6048 & 16 & 987136 & 104 & -48807306240 \\
7 & -16744 & 17 & -6905934 & $\ldots$ & $\ldots$ \\
8 & 84480 & 18 & 2727432 & 1050 & -2582248173523200 \\
9 & -113643 & 19 & 10661420 & 1051 & -56138975103853948 \\
10 & -115920 & 20 & -7109760 & $\ldots$ & $\ldots$ \\
\hline
\end{tabular}

For example, if we list the $\tau(n)$ modulo 23, then a certain pattern emerges:

\begin{tabular}{|c|c|c|c|c|c|c|c|}
\hline$n$ & $\tau(n)(\bmod 23)$ & $n$ & $\tau(n)$ & $(\bmod 23)$ & $n$ & $\tau(n)$ & $(\bmod 23)$ \\
\hline 1 & 1 & 11 & & 0 & $\ldots$ & & $\ldots$ \\
\hline 2 & -1 & 12 & & 0 & 100 & & 0 \\
\hline 3 & -1 & 13 & & -1 & 101 & & 2 \\
\hline 4 & 0 & 14 & & 0 & 102 & & 0 \\
\hline 5 & 0 & 15 & & 0 & 103 & & 0 \\
\hline 6 & 1 & 16 & & -1 & 104 & & -1 \\
\hline 7 & 0 & 17 & & 0 & $\ldots$ & & $\ldots$ \\
\hline 8 & 1 & 18 & & 0 & 1050 & & 0 \\
\hline 9 & 0 & 19 & & 0 & 1051 & & -1 \\
\hline 10 & 0 & 20 & & 0 & $\ldots$ & & $\ldots$ \\
\hline
\end{tabular}

Examination of this data leads to the conjecture $\tau(n) \equiv 0(\bmod 23)$ if $n$ is coprime to 23 and is quadratic nonresidue modulo 23. This was proven by Ramanujan via an identity of Euler.

The following congruences mentioned earlier can also be found in his notes:

$$
\begin{aligned}
\tau(n) & \equiv \sigma_{11}(n) \quad\left(\bmod 2^{8}\right) \quad(\text { for } n \text { odd }) \\
\tau(n) & \equiv n^{2} \sigma_{7}(n) \quad\left(\bmod 3^{3}\right) \\
\tau(n) & \equiv n \sigma_{9}(n) \quad\left(\bmod 5^{2}\right) \\
\tau(n) & \equiv n \sigma_{3}(n) \quad(\bmod 7), \\
\tau(n) & \equiv \sigma_{11}(n) \quad(\bmod 691) .
\end{aligned}
$$

To illustrate the 691 congruence we have calculated several coefficients modulo 691 . Obviously, one has to have some other insight to guess the congruence of $\tau(n)$ with $\sigma_{11}(n)$. 


\begin{tabular}{|c|c|c|c|c|c|c|c|}
\hline$n$ & $\tau(n)(\bmod 691)$ & $n$ & $\tau(n)$ & $(\bmod 691)$ & $n$ & $\tau(n)$ & $(\bmod 691)$ \\
\hline 1 & 1 & 11 & & 469 & $\ldots$ & & $\ldots$ \\
\hline 2 & 667 & 12 & & 123 & 100 & & 398 \\
\hline 3 & 252 & 13 & & 629 & 101 & & 210 \\
\hline 4 & 601 & 14 & & 385 & 102 & & 140 \\
\hline 5 & 684 & 15 & & 309 & 103 & & 539 \\
\hline 6 & 171 & 16 & & 388 & 104 & & 20 \\
\hline 7 & 531 & 17 & & 611 & . & & $\ldots$ \\
\hline 8 & 178 & 18 & & 55 & 1050 & & 67 \\
\hline 9 & 372 & 19 & & 672 & 1051 & & 464 \\
\hline 10 & 168 & 20 & & 630 & & & \\
\hline
\end{tabular}

Let $D \in \mathbb{Z}$ and

$$
N_{a}(D):=\#\left\{x \quad(\bmod 2 a) \mid x^{2} \equiv D \quad(\bmod 4 a)\right\} .
$$

Then put

$$
L_{D}(s):=\frac{\zeta(2 s)}{\zeta(s)} \sum_{a=1}^{\infty} N_{a}(D) a^{-s} .
$$

This function has a meromorphic continuaton to the whole complex plane and can be evaluated at negative integers. The generalized class numbers of Cohen are

$$
H(r, N):=L_{-N}(1-r)
$$

for $r \in \mathbb{N}$ odd and $N \in \mathbb{N}_{0}$.

Theorem 1.2. For all positive integers $n$ we have congruences between the Ramanujan numbers $\tau(n)$ and the generalized class numbers $H(11, N)(0<N \leq 4 n)$ with respect to the irregular prime numbers 131 and 593. Then we have:

$$
\tau(n) \equiv \frac{H(11,4 n)+2 \sum_{\substack{0<-D<4 n \\ D+4 n=\square}} H(11,-D)}{H(11,4)+2 H(11,3)} \quad(\bmod q) .
$$

For example, from the data here,

$$
\begin{aligned}
\tau(3) & \equiv \frac{H(11,12)+2 H(11,11)+2 H(11,8)+2 H(11,3)}{H(11,4)+2 H(11,3)} \quad(\bmod 131) \\
& \equiv \frac{-7750665838 / 3-2 \cdot 1035686046-2 \cdot 36581523-2 \cdot 3694 / 3}{-50521 / 2-2 \cdot 3694 / 3} \quad(\bmod 131) .
\end{aligned}
$$

The first $n \in \mathbb{N}$ such that $\tau(n) \equiv 0(\bmod 131)$ is given by $n=1051$. This we found with Pari. From the properties of $\tau(n)$ already mentioned it follows that 1051 has to be a prime. But more is true, since 131 is not an exceptional prime. That is, Deligne's results on Galois representations attached to modular forms can be applied, leading to congruences of generalized class numbers. For the irregular primes 131 and 593 we have congruences satisfied for a set of primes of positive density and restricted to an arbitary arithmetic progression. 
Theorem 1.3. Let $a, b$ and $l$ be positive integers and let $a$ and $l$ be coprime. Assume

$$
\mathbb{X}_{l}(a):=\{p \text { prime } \mid p \equiv a \quad(\bmod l)\}
$$

Then the congruence

$$
H(11,4 p)+2 \sum_{\substack{0<-D<4 p \\ D+4 p=\square}} H(11,-D) \equiv b \quad(\bmod 131 \cdot 593)
$$

is satisfied by a subset of $\mathbb{X}_{l}(a)$ of positive density.

\section{Generalized Class numbers, Galois representation AND CONGRUENCES}

It is well known that the discriminant $\Delta$ is an elliptic cusp form. This makes applicable the theory of modular forms and properties of the associated Galois representation.

For a positive even integer $k$, let $S_{k}$ be the modular forms of weight $k$ with respect to $S L_{2}(\mathbb{Z})$. A primitive newform $f \in S_{k}$ has $a_{1}=1$ and is an eigenform for Hecke operators. Let $x$ and $y$ be the real and imaginary part of $z$. The Petersson norm of $\Delta$ is given by

$$
\sqrt{\int_{S L_{2}(\mathbb{Z}) \backslash \mathfrak{H}} \Delta(z) \overline{\Delta(z)} \operatorname{Im}(z)^{10} d x d y}
$$

In the paper [1, H. Cohen introduced generalized class numbers $H(t, N)$, where $t \in \mathbb{N}$ and $-N$ is the discriminant of a binary quadratic form. Specifically, the numbers $H(1, N)$ are the Hurwitz-Kronecker class numbers $H(n)$, defined for $N>$ 0 . Let $t$ be odd and $D:=-N<0$. Let $D=D_{0} f^{2}$, where $D_{0}$ is a discriminant of a quadratic number field. Then

$$
H(t,-D):=L\left(1-t, \chi_{D_{0}}\right) \sum_{d \mid f} \mu(d) \chi_{D_{0}}(d) d^{t-1} \sum_{l \mid(f / d)}\left(\frac{f}{d}\right)^{2 t-1}
$$

Here $L\left(s, \chi_{D_{0}}\right)$ is the Dirichlet series $\sum_{n=1}^{\infty} \chi_{D_{0}}(n) n^{-s}$ attached to the Dirichlet character $\left(\frac{D}{*}\right)$ and $\mu$ the Moebius function. For $D=0$ put $H(t, 0)=\zeta(1-2 t)$. The values of the Riemann zeta function $\zeta(s)$ at negative odd negative integers $s=1-m$ are given by $-\frac{B_{m}}{m}$ where $B_{m}$ is the $m$-th Bernoulli number. It is not difficult to see that this coincides with the definition of $H(t, N)$ given in the introduction.

Then $H(t, N)$ is a rational number and for fixed odd $t_{0}>0$ the denominator of $H\left(t_{0}, N\right)$ is bounded. Cohen gave numerical data in the case $t=1,2,3,4$, extended in the book of Eichler and Zagier towards $t=5,7$ (for Jacobi forms). Since the Ramanujan numbers $\tau(n)$ are directly related to the case $t=11$ we have calculated 
$H(11, N)$ for $N=3,4 \ldots, 35$, and also the residues modulo 131 and 593:

\begin{tabular}{|r|r|c|c|}
\hline$N$ & $H(11, N)$ & $(\bmod 131)$ & $(\bmod 593)$ \\
\hline \hline 3 & $-3694 / 3$ & 35 & 350 \\
\hline 4 & $-50521 / 2$ & 88 & 535 \\
\hline 7 & -9006448 & 64 & 36 \\
\hline 8 & -36581523 & 96 & 54 \\
\hline 11 & -1035686046 & 85 & 314 \\
\hline 12 & $-7750665838 / 3$ & 84 & 238 \\
\hline 15 & -26914406416 & 119 & 350 \\
\hline 16 & $-1059502667113 / 2$ & 70 & 100 \\
\hline 19 & -3217455764142 & 74 & 545 \\
\hline 20 & -551609685150 & 86 & 40 \\
\hline 23 & $-55067979610704 / 23$ & 27 & 392 \\
\hline 24 & -3741386059246 & 60 & 199 \\
\hline 27 & $-38640544735576 / 3$ & 4 & 471 \\
\hline 28 & -18878676839792 & 3 & 208 \\
\hline 31 & -54994838246496 & 30 & 114 \\
\hline 32 & -76717050704019 & 124 & 459 \\
\hline 35 & -196480469755548 & 67 & 527 \\
\hline
\end{tabular}

Proof of Theorem 1.3. Let $q=131$ or 593. From Table (2.3)

$$
H(11,4)+2 H(11,3) \quad(\bmod q)
$$

is a unit $\kappa_{q}$ in the field $\mathbb{Z} / q \mathbb{Z}$. This allows us to transfer a result of Swinnerton-Dyer [12] on elliptic modular forms, applied to $\Delta$ and combined with Theorem 1.2, to the generalized class numbers. Let $f \in S_{k}$ be a primitive newform with the property that all Fourier coefficients are rational integers. Then the following is satisfied: Let $m_{1}$ and $m_{2}$ be positive integers, and let $c \in \mathbb{Z} / m_{1} \mathbb{Z}$ and $d \in\left(\mathbb{Z} / m_{2} \mathbb{Z}\right)^{*}$. Suppose that any prime divisor of $m_{1}$ is not exceptional for $f$. Then the set of prime numbers $p$ such that $p \equiv d\left(\bmod m_{2}\right)$ with the property $a_{p} \equiv c\left(\bmod m_{1}\right)$ has positive density.

Since the discriminant function $\Delta$ is a normalized cusp form of weight 12 and $\operatorname{dim} S_{12}=1$, the function $\Delta$ is a primitive newform. Moreover, it follows from the definition that all Fourier coefficients are integers. Since $\{2,3,5,7,23,691\}$ is the set of all exceptional primes related to $l$-adic Galois representations of $\Delta$ we can apply the theorem for the case $m_{1}=131$ and 593 .

\section{The Ramanujan numbers and the Symmetric SQuare $L$-SERIES}

The symmetric square $L$-function of $\Delta$ is

$$
D(\Delta, s)=\frac{\zeta(2 s-22)}{\zeta(s-11)} \sum_{n=1}^{\infty} \tau(n)^{2} n^{-s} .
$$

It converges absolutely for $\operatorname{Re}(\mathrm{s})>12$ and has a holomorphic continuation to the complex plane. Further, $m=22$ is a critical value in the sense of Deligne, and

$$
D(\Delta, 22) / \pi^{11}=\|\Delta\|^{2} Q
$$

with $Q \in \mathbb{Q}^{*}$ and $\|\Delta\|$ the Petersson norm of $\Delta$. 
3.1. Proof of Theorem 1.1. For $g, k$ positive integers and $k>g+1$ denote by $E_{k}^{g}(z)$ the holomorphic Siegel-Eisenstein series of weight $k$ on the Siegel upper half-space $\mathfrak{H}_{g}$,

$$
E_{k}^{g}(z):=\sum_{\left(\begin{array}{ll}
a & b \\
c & d
\end{array}\right) \in \Gamma_{\infty} \backslash \Gamma_{g}} \operatorname{det}(c z+d)^{-k},
$$

where $\Gamma_{g}=S p_{g}(\mathbb{Z})$ is the Siegel modular group and $\Gamma_{\infty}=\left\{\left(\begin{array}{ll}a & b \\ c & d\end{array}\right) \in \Gamma_{g} \mid c=0\right\}$. The Fourier coefficients of the Eisenstein series,

$$
E_{k}^{g}(z)=\sum_{T \in X_{g}} A_{k}^{g}(T) e^{2 \pi i \operatorname{tr}(T z)}
$$

( $X_{g}$ the set of half-integral positive semi-definite matrices) are rational integers with bounded denominator. We are interested in $g=1$ and $g=2$. The classical Eisenstein series $E_{k}(z)=E_{k}^{1}(z)$ of degree one has the Fourier expansion

$$
E_{k}(z)=1+\frac{-2 k}{B_{k}} \sum_{n=1}^{\infty} \sigma_{k-1}(n) q^{n} \quad\left(q=e^{2 \pi i z}\right),
$$

$\sigma_{m}(n):=\sum_{d \mid n} d^{m}$. The Bernoulli numbers can be defined by

$$
\frac{x}{e^{x}-1}=\sum_{n=0}^{\infty} B_{n} \frac{x^{n}}{n !}
$$

For example,

$$
B_{0}=1, B_{1}=-\frac{1}{2}, B_{2}=\frac{1}{6}, B_{3}=0, B_{4}=-\frac{1}{30}, B_{6}=\frac{1}{42}, B_{8}=-\frac{1}{30}, \ldots .
$$

It is well known that $B_{m}=0$ for $m>1$ odd. From Kummer's study of Fermat's Last Theorem we have the concept of irregular primes, and all primes $p$ dividing the numerator $\mathcal{N}_{k}$ of $\frac{B_{k}}{-2 k}$ are irregular. We will show that the Ramanujan numbers $\tau(n)$ are related to $\mathcal{N}_{12}=691$. The arithmetic nature of the Fourier coefficients of the Eisenstein series of degree 2 is more complicated but we have still some control. With $(n, r, m):=\left(\begin{array}{cc}n & \frac{r}{2} \\ \frac{r}{2} & m\end{array}\right) \in X_{2}$, the denominator of the rational integer

$$
\mathcal{N}_{k} \mathcal{N}_{2 k-2} A_{k}^{2}(n, r, m)
$$

is not divisible by $p \mid \mathcal{N}_{k} \mathcal{N}_{2 k-2}$. Maass conjectured that such primes $p$ are optimal, in the sense that, for $p \mid \mathcal{N}_{k} \mathcal{N}_{2 k-2}$ there exists a $T \in X_{2}$ such that

$$
\mathcal{N}_{k} \mathcal{N}_{2 k-2} A_{k}^{2}(T) \in \mathbb{Z}_{p}^{*}
$$

To prove the 691 Ramanujan congruences for $\tau(n)$ it is sufficient that

$$
691^{2} A_{12}^{2}(T) \equiv 0 \quad(\bmod 691) .
$$

But this is well known and follows from results of Maass 8. Let $D<0$ be a discriminant. Then for all $T=(n, r, 1) \in X_{2}$ with $D=r^{2}-4 n$,

$$
A_{k}^{2}(n, r, 1)=2 \frac{L_{D}(2-k)}{\zeta(1-k) \zeta(3-2 k)} \text {. }
$$


Further, we have $A_{k}^{2}(0,0,0)=0$ and $A_{k}^{2}(n, 0,0)=A_{k}^{1}(n)$. The calculation of these rational integers is straightforward. For example,

$$
\begin{aligned}
& A_{12}^{2}(1,0,1)=\frac{2^{5} \cdot 3^{3} \cdot 5 \cdot 7 \cdot 13 \cdot 19 \cdot 23 \cdot 2659}{131 \cdot 593 \cdot 691}, \\
& A_{12}^{2}(1,1,1)=\frac{2^{7} \cdot 3^{2} \cdot 5 \cdot 7 \cdot 13 \cdot 23 \cdot 1847}{131 \cdot 593 \cdot 691}, \\
& A_{12}^{2}(1,2,1)=\frac{2^{4} \cdot 3^{2} \cdot 5 \cdot 7 \cdot 13}{691} .
\end{aligned}
$$

Put $A_{12}^{2}(1,1):=A_{12}^{2}(1,0,1)+2 A_{12}^{2}(1,1,1)+2 A_{12}^{2}(1,2,1)$. Then

$$
A_{12}^{2}(1,1)=\frac{2^{8} \cdot 3^{4} \cdot 5^{2} \cdot 7^{2} \cdot 13 \cdot 1549}{131 \cdot 593 \cdot 691} .
$$

Fixing the imbedding $\mathfrak{H} \times \mathfrak{H}$ into $\mathfrak{H}_{2},(z, w) \mapsto\left(\begin{array}{cc}z & 0 \\ 0 & w\end{array}\right), E_{k}^{2}(z, w) \in \operatorname{Sym}^{2} M_{k}$, where $M_{k}$ denotes the space of elliptic modular forms of weight $k$ with respect to $\Gamma=S L_{2}(\mathbb{Z})$. Since $M_{12}=\mathbb{C} E_{12} \oplus \mathbb{C} \Delta$,

$$
\left.E_{12}^{2}\right|_{\mathfrak{H} \times \mathfrak{H}}=E_{12} \otimes E_{12}+\alpha\left(E_{12} \otimes \Delta+\Delta \otimes E_{12}\right)+\beta \Delta \otimes \Delta .
$$

It follows from [7 that $\alpha=0$ and $\beta \neq 0$, since Siegel type Eisenstein series are elements of the Maass Spezialschar and there are no elliptic cusp forms of weight 14. Moreover, it is obvious that $\beta$ is a rational number. Garrett's viewpoint [4], 5] on the doubling method and the triple $L$-function leads to

$$
\beta=\frac{\pi}{2^{9} \cdot 11 \cdot \zeta(12) \cdot \zeta(11)\|\Delta\|^{2}} \sum_{n=1}^{\infty} \tau(n)^{2} n^{-22} .
$$

The Fourier expansion and Fourier coefficients $A_{12}^{2}(n, m)$ of $\left.E_{12}^{2}\right|_{\mathfrak{H} \times \mathfrak{H}}$ can be easily deduced from $A_{12}^{2}(n, r, m)$, since

$$
A_{12}^{2}(n, m)=\sum_{r \in \mathbb{Z}, r \leq \sqrt{4 n m}} A_{12}^{2}(n, r, m) .
$$

Let $a(n)$ denote the Fourier coefficient of the elliptic Eisenstein series. Then we obtain from (3.14) the identity

$$
A_{12}^{2}(1,1)=a(1)^{2}+\frac{\pi}{2^{9} \cdot 11 \cdot \zeta(12) \cdot \zeta(11)\|\Delta\|^{2}} \sum_{n=1}^{\infty} \tau(n)^{2} n^{-22} .
$$

Then we have $691^{2} \beta \in \mathbb{Z}_{691}^{*}$ and $\frac{\beta}{a(1)^{2}} \equiv-1(\bmod 691)$. This proves the first part of the theorem on the quadratic residue mod 691 . We also have

$$
A_{12}^{2}(n, 1)=a(n) a(1)+\beta \tau(n) .
$$

Hence $0 \equiv \frac{a(n)}{a(1)}+(-1) \tau(n)(\bmod 691)$. This proves the Ramanujan congruence in the theorem since $a(n) / a(1)=\sigma_{11}(n)$.

Remark. Note that this proof is constructive. For example, it is easy to deduce that

$$
\beta=\frac{2^{14} \cdot 3^{7} \cdot 5^{3} \cdot 7^{3} \cdot 13}{131 \cdot 593 \cdot 691^{2}}
$$


3.2. Congruences between the Ramanujan numbers and class numbers. In this subsection we prove that $\tau(n)$ satisfies certain congruences mod 131 and 593 related to the class numbers $H(11, N)$. Here $\mathcal{N}_{22}=131 \cdot 593$.

Proof of Theorem 1.2. We have already seen that $A_{12}^{2}(1,1)=a(1)^{2}+\beta$. From this we easily obtain for $q=131$ or 593 the congruence

$$
2(H(11,4)+2 H(11,3)) \equiv \zeta(-11) \cdot \zeta(-21) \cdot \beta \quad(\bmod q) .
$$

Here we used the explicit formula of the Fourier coefficients of the Siegel type Eisenstein series and, twice, the fact that $\zeta(-11) \zeta(-21) a(1) \equiv 0(\bmod q)$. More generally, let $n \in \mathbb{N}$ and choose $r \in \mathbb{N}$ such that $4 n-r^{2}>0$ and $4 n-r^{2} \leq 0$. Then

$$
\begin{gathered}
2\left(H(11,4 n)+2 H(11,4 n-1)+\ldots+2 H\left(11,4 n-r^{2}\right)\right) \\
\equiv \zeta(-11) \cdot \zeta(-21) \cdot \beta \cdot \tau(n) \quad(\bmod q) .
\end{gathered}
$$

Since $H(11,4)+2 H(11,3) \not \equiv 0(\bmod q)$ the theorem is proven.

\section{ACKNOWLEDGEMENT}

The author thanks the referee for helpful comments and suggestions that improved the quality of this paper.

\section{REFERENCES}

[1] H. Cohen: Sums involving the values at negative integers of L-functions of quadratic characters. Math. Ann. 217, (1975) 271-285. MR0382192 (52:3080)

[2] P. Deligne: Formes modulaires et representations l-adiques. Seminaire Bourbaki No. 355 (1969).

[3] M. Eichler, D. Zagier: The theory of Jacobi forms. Progress in Mathematics. Vol. 55. BostonBasel-Stuttgart: Birkhäuser (1985). MR.781735 (86j:11043)

[4] P. Garrett: Decomposition of Eisenstein series: triple product $L$-functions. Ann. Math. 125 (1987), 209-235. MR881269 (88m:11033)

[5] P. Garrett: On the arithmetic of Siegel-Hilbert cuspforms: Petersson inner products and Fourier coefficients. Invent. Math. 107 (1992), 453-481. MR.1150599(93e:11060)

[6] G.H. Hardy: Ramanujan. Twelve lextures on subjects suggested by his life and his work. Cambridge Univ. Press (1940). MR0004860 (3:71d)

[7] B. Heim: On the Spezialschar of Maass. Preprint, submitted 2006

[8] H. Maass, H.: Über die Fourierschen Koeffizienten von Eisenstein Reihen zweiten Grades. Mat. fys. Med. Danske Vid. Selsk. 34 (1964). MR0171758 (30:1985)

[9] K. Ono: Congruences on the Fourier coefficients of modular forms on $\Gamma_{0}(N)$. Contemporary Mathematics, Vol. 166, (1994) 93-105. MR:1284053 (95g:11038)

[10] J.-P. Serre: Abelian l-adic representations and elliptic curves. New York (1968). MR0263823 $(41: 8422)$

[11] S. Ramanujan: On certain arithmetical functions. Trans. Cambridge Phil. Soc. 22 (1916), 159-184.

[12] J.-P. Serre: Congruences et formes modulaires (d'apres Swinnerton-Dyer). Seminaire Bourbaki No. 416 (1971).

[13] D. Zagier: Modular forms whose Fourier coefficients involve zeta-functions of quadratic fields. Modular functions of one variable, VI (Proc. Second Internat. Conf., Univ. Bonn, Bonn, 1976), pp. 105-169. Lecture Notes in Math., Vol. 627, Springer, Berlin, 1977. MR0485703 $(58: 5525)$

MaX-Planck Institut für Mathematik, Vivatsgasse 7, 53111 Bonn, Germany

E-mail address: heim@mpim-bonn.mpg.de 